\title{
Maternal anemia - an important determinant of low birth weight babies - An observational study from central India
}

\author{
Jyotsna Verma', Sapna Jain ${ }^{2, *}$, Shweta Anand ${ }^{3}$ \\ ${ }^{1}$ Associate Professor, ${ }^{3}$ Professor, ${ }^{1,3}$ Dept. of Pediatrics, L.N. Medical College, Bhopal, Madhy a Pradesh, ${ }^{2}$ Associate Professor, \\ Dept. of Obstetrics \& Gynaecology, L.N. Medical College, Bhopal, Madhy a Pradesh, India
}

*Corresponding Author:

Email: sapna.bajaj48@yahoo.com

\begin{abstract}
Introduction: A baby's weight at birth is a strong indicator of maternal and newborn health and nutrition. Among the various factors affecting the birth weight, anemia during pregnancy still has been found to have a significant effect on weight as well as maturity of the baby. ${ }^{1}$

Objective: Present study is an attempt to find out the impact of corrected maternal anemia on the perinatal outcome, assessed in terms of birth weight and gestational age in comparison to babies born to mothers with anemia during pregnancy and at the time of delivery.

Materials and Methods: Total 144 pregnant women of $20 \pm 4$ weeks of gestation with singleton pregnancy attending OPD of Department of Obstetrics \& Gynaecology from January 2015 to December 2015 were enrolled in our study. Antenatal mothers with hemoglobin level between 7-10 gm\% constituted our study group, and were supplemented with hematinics. Perinatal outcome in terms of birth weight and gestational age was assessed in babies of mother with improved anemia and compared with babies delivered to mother with moderate anemia at the time of delivery.

Results: Out of 144 registered 63.8\% women had moderate anemia, which constituted our study group. On supplementation with hematinics to these antenatal mothers, improvement in mean hemoglobin was reported to be from $8.4 \mathrm{gm} \%$ to $11.4 \mathrm{gm} \%$. On comparing Perinatal outcome of this group with babies of anaemic mother, there was statistically significant increase in low birth weight babies of $27.8 \%(\mathrm{p}<0.0023)$. No significant impact of maternal anemia on gestational age was appreciated in our study.

Conclusion: Anemic mothers are at increased risk of delivering low birth weight babies, which again is an important determinant of survival and quality of life of a child. Positive impact of improvement of maternal anemia on birth weight of babies can be considered as an effective antenatal intervention taken to improve health of our newborns and their mothers.
\end{abstract}

Keywords: Maternal anemia, Perinatal outcome, Premature and low birth weight babies.

\section{Introduction}

Madhya Pradesh is one of the states of India having high infant mortality rate, as well as malnutrition. ${ }^{2,3}$ Birth weight of a baby is one of the important determinants of child nutrition and health. Birth weight in turn is determined to a great extent by factors that operate in utero, well before they are born. ${ }^{4}$ Low birth weight babies often face severe immediate consequences in terms of increased mortality, morbidity and disability and long term health consequences in the form of increased susceptibility to adulthood diseases like diabetes and hypertension.

There is significant variation in the incidence of LBW across regions. In 2013, nearly 22 million newborns an estimated $16 \%$ of all babies born globally had low birth weight. South Asia has the highest incidence, with one in four newborns weighing less than $2500 \mathrm{gms}$, while East Asia/Pacific has the lowest, at $7 \%$. Nearly $40 \%$ of all LBW babies in the developing world are born in India. ${ }^{6}$

Among the various factors affecting the birth weight, anemia during pregnancy has been found to have significant affect on weight as well as on maturity of baby. WHO estimates that prevalence of anemia is $14 \%$ in developed countries, $42.7-57.3 \%$ in developing countries like India. ${ }^{7}$
According to NFHS- 4 and various studies, percentage of women suffering from anemia during pregnancy ranges from 54.6 to $70 \% .^{3,7-9}$ Multiple factors lead to anaemia in pregnancy, nutritional iron deficiency anaemia (IDA) being the commonest. Physiological haemodilution and several factors affecting $\mathrm{Hb}$ and iron status in pregnancy lead to difficulties in establishing a definitive diagnosis. Fetal programming occurs when the normal pattern of fetal development is disrupted by an abnormal stimulus or 'insult' applied at a critical point in inutero development. ${ }^{10}$ Correction of anaemia with haematinics provides adequate proof of a nutritional deficiency in its pathogenesis. Present study is an attempt to assess impact of corrected maternal anemia on perinatal outcome in terms of birth weight and gestational maturity and its comparison with babies born to anemic mothers and burden and severity of anemia in pregnant women attending OPD of Dept. of Obstetrics \& Gynaecology L.N. Medical College and J.K. Hospital Bhopal M.P.

\section{Methodology}

Setting and Samples: Study was conducted at Department of Pediatrics and Obstetrics and Gynaecology of L.N Medical College Bhopal after 
institutional ethical clearance. Total 144 pregnant women of $20 \pm 4$ weeks of gestation with singleton pregnancy attending OPD of Department of Obstetrics \& Gynaecology at J.K.Hospital L.N. Medical College from January 2015 to December 2015 were enrolled after taking informed consent. A brief medical history and examination was done before enrolling the patient.

Exclusion Criteria: Antenatal mothers having associated conditions that may affect the fetal growth were excluded

1. Women with systemic disease like primary cardiac, renal, hepatic disorder and hypothyroidism.

2. History of pathologic blood loss at any stage during study period

3. Antenatal mother who developed PIH even after enrollment.

4. Those that did not show improvement of anemia after supplementation.

5. Mothers who had anemia other than nutritional.

Laboratory Investigation: To know the severity of anemia complete haemogram was done, and as per WHO criteria, on the basis of hemoglobin leve $1^{11}$ they were divided into 3 groups

Group A $(\mathrm{Hb}<7)$ severe anemia

Group B ( $\mathrm{Hb} 7$ - 10) moderate anemia

Group $\mathrm{C}(\mathrm{Hb}>10)$ mild/ no anemia

Women of Group B with hemoglobin levels of 7$10 \mathrm{gm} \%$ became our study group. These were supplemented with oral hematinics as per protocol of management of anemia during pregnancy and these were followed till delivery for assessment of perinatal outcome. Oral haematinic was given in form of tablets with following composition Ferrous Ascorbate equivalent to elemental iron $100 \mathrm{mg}$, Folic acid $1.1 \mathrm{mg}$. These tablets were given one tablet twice daily after meals as per standard protocol of management of anemia during pregnancy. These patients were regularly followed till the time of delivery and improvement of anemia was assessed by hemoglobin levels done at regular intervals and at the time of delivery. Perinatal outcome in terms of prematurity and low birth weight babies was assessed in babies of mother who showed improvement of their anemia during follow up. Gestational age was assessed by modified Ballard scoring system (Ballard et al, 1991). Birth weight of babies of mothers enrolled for the study was taken by same digital weighing machine used in labor room. Low birth weight baby was defined as per standard definition of birth weight of less than $2500 \mathrm{gms}$.
Babies of this group were compared with babies of 79 mothers who had anemia at the time of delivery. Their records were checked retrospectively to assess their anemia status during pregnancy. Only those mothers were included in comparison group, whose hemoglobin levels were between $7-10 \mathrm{~g} \mathrm{~m} \%$ at the time of delivery and who had previous lab records suggestive of moderate anemia. Those mothers who were anemic but had any other risk factor which was likely to affect the growth of the baby were excluded.

\section{Result}

A total of 144 wo men with pregnancy around $20 \pm 4$ week's gestation were included in this study. As per hemoglobin levels and WHO definition of anemia, seven $(4.8 \%)$ antenatal mothers had severe anemia, ninety two $(63.8 \%)$ had moderate anemia and $45(31 \%)$ had $\mathrm{Hb}$ levels $>10 \mathrm{~g} \mathrm{~m} / \mathrm{dl}$ (Table 1$)$. Ninety two women with moderate anemia i.e. he moglob in level between 7 $10 \mathrm{gm} \%$ constituted ourstudy group.

Out of 92 women 3 were lost to follow up, one who developed PIH, one with bleeding and 9 who did not show improvement in hemoglobin level due to irregular intake or intolerance were excluded from this study.

In remaining 78 women improvement in mean hemoglobin was reported to be from $8.4 \mathrm{~g} \mathrm{~m} \%$ to 11.4 gm\%. Perinatal outcome in terms of birth weight and gestational age was assessed in these 78 women and was compared with 79 babies delivered to anemic mothers. Anemia status of two groups as per their hemoglobin levels is depicted in Table 2. Improvement of maternal anemia was found to have significant positive effect on birth weight of baby in our study. Seven $(8.9 \%)$ babies had low birth weight in supplemental group as compared to $27.8 \%$ in anemic group (Table 3)

Mean birth weight was $2746 \mathrm{gms}$ in non anemic group (supplemental group) as compared to $1876 \mathrm{gms}$ in anemic group. Babies of non anemic group showed improvement in birth weight of about $870 \mathrm{gms}$ with maternal supplementation (Table 4).

Effect of improvement of maternal anemia on maturity of baby is shown in Table 5. No significant effect of maternal anemia on gestational age could be demonstrated in our study.

Table 1: Distribution of antenatal mothers according to their hemoglobin level

\begin{tabular}{|c|c|c|c|c|}
\hline Groups & Range of Hb $(\mathrm{gm} / \mathrm{dl})$ & Study group $\mathrm{n}=144$ & Mean Hb. $(\mathrm{gm} / \mathrm{dl})$ & HCT \\
\hline $\mathrm{A}$ & $<7$ & $7(4.8 \%)$ & 6.4 & 19.2 \\
\hline $\mathrm{B}$ & $7-10$ & $92(63.8 \%)$ & 8.6 & 25.8 \\
\hline $\mathrm{C}$ & $>10$ & $45(31.2 \%)$ & 11.6 & 34.8 \\
\hline
\end{tabular}


Table 2: Anemia status of antenatal mothers at the time of delivery

\begin{tabular}{|l|c|c|}
\hline \multicolumn{1}{|c|}{ Groups } & Supplemented Group (78) & Comparison Group (79) \\
\hline $\begin{array}{l}\text { Mean Hb Level (gm/dl) } \\
\text { Before supplementation }\end{array}$ & $\sim 8.4$ & \\
\hline $\begin{array}{l}\text { Mean Hb level (gm/dl) } \\
\text { After supplementation }\end{array}$ & $\sim 11.4$ & $\sim 8.2$ \\
\hline HCT & 34.2 & 24.6 \\
\hline
\end{tabular}

Table 3: Distribution of babies of anemic and non-anemic mothers as per their birth weight

\begin{tabular}{|c|c|c|c|}
\hline Groups & $\begin{array}{c}\text { Antenatal } \\
\text { mothers }(\mathbf{n})\end{array}$ & $\begin{array}{c}\text { Birth Weight }(>2500 \text { gms }) \\
\text { Normal weight babies }\end{array}$ & $\begin{array}{c}\text { Birth Weight <2500 gms } \\
\text { Low birth weight babies }\end{array}$ \\
\hline Non Anemic mothers & 78 & $71(91 \%)$ & $7(8.9 \%)$ \\
\hline Anemic mothers & 79 & $57(72 \%)$ & $22(27.8 \%)$ \\
\hline
\end{tabular}

Chi squared equals 9.284 with 1 degrees of freedom. The two-tailed $\mathrm{P}$ value equals $0.0023(\mathrm{P}<0.05)$

Table 4: Mean birth weight in two groups

\begin{tabular}{|c|c|}
\hline Groups & Mean Birth wt (gms) \\
\hline Non anemic group & 2746 \\
\hline Anemic group & 1876 \\
\hline
\end{tabular}

Table 5: Distribution of babies according to their gestation in anemic and non anemic group

\begin{tabular}{|l|c|c|c|c|}
\hline \multicolumn{1}{|c|}{ Groups } & Preterm & Term & $\begin{array}{c}\text { Post } \\
\text { term }\end{array}$ & Total \\
\hline $\begin{array}{l}\text { Non anemic } \\
\text { group (78) }\end{array}$ & $5(6.4 \%)$ & 72 & 1 & 78 \\
\hline $\begin{array}{l}\text { Anemic } \\
\text { Group (79) }\end{array}$ & $9(11.3 \%)$ & 70 & 0 & 79 \\
\cline { 1 - 4 } & & &
\end{tabular}

Chi-square without Yates correction Chi squared equals 1.199 with 1 degrees of freedom

The two-tailed $\mathrm{P}$ value equals 0.2734

\section{Discussion}

Maternal Anemia: Prevalence of maternal anemia in India as per NFHS 3 was reported to be $\sim 58 \%$ which has not changed much, NFHS 4 reports it to be $\sim 55 \%$. Many studies from different parts of India have reported prevalence of anemia during pregnancy ranging from as low as $31.65 \%$ from Karnataka 14 to as high as $87 \%$ by Kalaivani K. ${ }^{12,13}$ In our study $\sim 70 \%$ of women were anemic of which $\sim 68 \%$ had moderate to severe anemia. Manisha et $\mathrm{Al},{ }^{14}$ Githartha bordoloi ${ }^{15}$ and Ekta dalal ${ }^{8}$ from central India also found similar prevalence of maternal anemia in their studies.

Low Birth Weight Babies: In India, of all infants who died before they completed 29 days post-birth, $48.1 \%$ suffered from LBW and premature birth. ${ }^{16,17}$ Maternal anemia is reported to be one of the important factors of low birth weight of babies. The effect of maternal anemia on birth weight have been reported by many studies, but the level of hemoglobin at which this association is seen is reported to be, different in various studies. Most of the studies reported that hemoglobin level of less than $10 \mathrm{~g} \mathrm{~m} \%$ had significant effect on birth weight of baby ${ }^{13,16-18}$ but Manisha et $\mathrm{al}^{14}$ found positive association only with severe anemia with hemoglobin level $<7 \mathrm{gm} \%$. Similar inverse relation with maternal hemoglobin level between 6-8 gm\% and low birth weight was reported by Ganesh et al. ${ }^{19}$ In contrast $\mathrm{K}$. Jagdish et al in their study observed no association between maternal anemia and birth weight of babies. ${ }^{20}$

In our study percentage of low birth weight babies was $27.8 \%$ in anemic mothers which was high as compared to $8.9 \%$ in non anemic mothers. The overall prevalence of LBW as reported by various studies from India ranged from $28 \%$ to $40 \%$. $^{12,17,19,20}$

Risk of having low birth weight baby increases when mother is anemic. ${ }^{21,22}$ It was 1.9 to 4.8 times more as reported by Lone FW and Deshmukh.et al in their study. ${ }^{23,25}$

Maternal ane mia has long been a known risk factor for preterm delivery along with its effect on birth weight. In our study $6.4 \%$ babies were born prematurely to non anemic mothers as compared to $11.3 \%$ babies of anemic mothers. Incidence of preterm delivery was reported to be as high as $45 \%$ in moderate anemia group as compared to $50 \%$ in severely anemic mothers. ${ }^{26}$ In our study intrauterine growth retardation was more responsible for low birth weight than prematurity. On the contrary Sharma $\mathrm{S}$ reported prematurity to be more associated with low birth weight. $^{27}$

\section{Conclusion}

From the present study we conclude that; anemic mothers had higher chance of delivering preterm and low birth weight baby, which again is an important determinant of survival and quality of life a child. This reiterates importance of preventing and controlling maternal anemia not only for mothers but also for the health of a baby.

\section{References}

1. Global Nutrition Targets 2025: Low birth weight policy brief. www.who.int/nutrition/publications/globaltargets2025_po licybrief_lbw/en/

2. State/UT -wise Infant Mortality Rate during 2015-16. https://community.data.gov.in

3. State fact sheet Madhya Pradesh -District level household survey Rchiips.org/nfhs/pdf/nfhs4/mp_factsheet. 
4. Myatt L. Placental adaptive responses and fetal programming. J Physiol. 2006 Apr 1;572(1):25-30.

5. Barker DJP, Osmond C, Kajantie E, Eriksson J. Growth and chronic disease: findings in the Helsinki Birth Cohort. Ann Hum Biol.2009;36:445-58.

6. Low birthweight-UNICEF DAT A. https://data.unicef.org.

7. Global Health Observatory (GHO) - World Health Organization http://www.who.int/gho/about/en/.

8. Kumar.et al. Maternal anemia in various trimesters and its effect on newborn weight and maturity: An observational study Int J Prev Med. 2013 Feb; 4(2):193-199.

9. Dalal E, Shah J. A comparative study on outcome of neonates born to anemic mothers versus non anemic mothers. Natl J Med Res. 2014;4(4):270-3.

10. H.K.Cheema, Bajwa B S, Kaur K, Joshi H. Prevalence and possible risk factor of anemia in different trimester of pregnancy. International Journal of Contemporary Medical Research 2016;3(4):1194-1197.

11. Haemoglobin concentrations for the Diagnosis of AnaeWorld Health...2011.

www.who.int/vmnis/indicators/haemoglobin/en.

12. Anjanappa B, Radhika BH, Nataraja HG, Renuka R, Sathya P. Maternal haemoglobin and perinatal outcome. Int J Reprod Contracept Obstet Gynecol. 2015 Oct;4(5):1335-1338

13. K. Kalaivani. Prevalence \& consequences of anaemia in pregnancy. Indian J Med Res 130, November 2009, pp 627-633.

14. Manisha N, Choudhury M K, Choudhury S S, Kakoty S D, Sarma U C, Webster P et.al. Association between maternal anaemia and pregnancy outcomes: a cohort study in Assam, India. BMJ Glob Health.2016;1(1):e000026

15. Bordoloi G. A. study on the relationship between hemoglobin concentration in third trimester of pregnancy and birth weight of the baby. Innovative Journal of medical and health science. 2017;7 p18-20.

16. Causes of death lbw govt.census report_2010-2013 release

17. Press Information Bureau Government of India Ministry of Health and Family Welfare 06-May-2016 14:18 IST Maternal and Child Mortality Rate.

18. Sekhavat.L, Davar R, Hosseinidezoki S. Relationship between maternal hemoglobin concentration and neonatal birth weight. Hematology.2011 Nov;16(6):373-6.

19. Ganesh K S, Harsha Kumar HN, Jayaram S and Kotian M S. Determinants of Low Birth Weight: A Case Control Study in a district hospital Karnataka Indian J Pediatr 2010; 77 (1) 87-89.

20. K Jagadish Kumar, N Asha, D Srinivasa Murthy, MS Sujatha, ${ }^{2}$ and VG Manjunath. Maternal Anemia in Various Trimesters and its Effect on Newborn Weight and Maturity: An Observational Study: Int J Prev Med. $2013 \mathrm{Feb} ; 4(2): 193-199$.

21. Gagan A, Sartaj A, Kapil G, Vijay Kumar, Parul G, et al. (2012) Maternal Risk Factors Associated with Low Birth Weight Neonates in a Tertiary Care Hospital, Northern India. J Community Med Health Educ 2:177.

22. Agarwal K, Agarwal A, Agrawal V K, Agrawal P, Chaudhary V. Prevalence and determinants of "low birth weight" among institutional deliveries. Ann Nigerian Med 2011;5:48-5

23. Lone FW, Qureshi RN, Emmanuel F. Maternal anaemia and its impact on perinatal outcome in a tertiary care hospital in Pakistan. Et Mediterr Health J. 2004 Nov;10(6):801-7.
24. Chauhan M, Tomar S. Anaemia in Pregnancy a Prospective Observational Study in Tertiary Care teaching hospital of North India. Journal of medical science and clinical research.2013 Nov;1(4):195-198.

25. Kumar V, Deshmukh PR, Taywade M, Gupta SS. Magnitude and Correlates of Low Birth Weight at Term in Rural Wardha, Central India. Online J Health Allied Scs. 2016;15(1):2.

26. Bangal V B, Aher K, Bhosale K, Tuse H. Study of maternal and Perinatal outcome in moderate to severe degree iron deficiency anaemia in rural community. International journal of biomedical and advanced research. 2016;7(2):088-093.

27. Sharma SR, Giri S, Timalsina U, Bhandari SS, Basyal B, Wagle K, et al. (2015) Low Birth Weight at Term and Its Determinants in a Tertiary Hospital of Nepal: A CaseControl Study. 